\title{
DIABETIC PATIENTS WHO ARE AMATEUR DIVERS
}

\author{
Daria Ługowska ${ }^{1)}$, Tomasz Ługowski ${ }^{2)}$, Olga Krzywińska ${ }^{3)}$, Mariusz Kozakiewicz ${ }^{3)}$, Paweł Grzelakowski ${ }^{2)}$ \\ 1) Clinic of Internal Diseases and Diabetology 10th Military Research Hospital with Polyclinic in Bydgoszcz, Poland \\ ${ }^{2)}$ Clinic of Cardiology and Cardiothorasic Surgery 10th Military Research Hospital with Polyclinic in Bydgoszcz, Poland \\ 3) Department and Faculty of Food Chemistry of Nicolaus Copernicus University in Toruń Collegium Medicum in Bydgoszcz, Poland
}

\section{ABSTRACT}

Diabetes is an "epidemic of the 21st century" and it is estimated that in $20116.7 \%$ of Poland's population suffered from diabetes, one quarter of which was unaware of the disease. Such a high prevalence of diabetes mellitus results in an increasing number of physicians being confronted with the problem of qualifying patient with diabetes to partake in amateur sports, including diving. This publication presents the most important information concerning risk assessment, admission of diabetic patients to amateur diving and some proposed protocols of management before and during sport practice. Keywords: diabetes mellitus, recreational diving.

\section{ARTICLE INFO}

PolHypRes 2018 Vol. 64 Issue 3 pp. 39- 44

ISSN: 1734-7009 elSSN: 2084-0535

DOI: $10.2478 / p h r-2018-0017$

Pages: 6, figures: 0 , tables: 0

page www of the periodical: www.phr.net.pl

Publisher

Polish Hyperbaric Medicine and Technology Society

\section{Review article}

Submission date: $22.06 .2018 \mathrm{r}$.

Acceptance for print: 02.08.2018 r. 


\section{INTRODUCTION}

Diabetes mellitus (DM) is a group of metabolic diseases characterised by hyperglycaemia resulting from a defect in insulin secretion and/or activity. Chronic hyperglycaemia is associated with damage, dysfunction and failure of various organs, in particular eyes, kidneys, nerves, heart and blood vessels [1].

The incidence of diabetes is steadily increasing, it is referred to as the "epidemic of the 21st century". In 2000 there were about 150 million people suffering from diabetes in the world, and it is estimated that by 2035 this number will reach 600 million people. The results of the NATPOL study showed that in 2011, 6.7\% of the Polish population suffered from diabetes, with a quarter of this population unaware of the disease [2].

Such a high prevalence of diabetes poses a challenge to health care systems around the world. Rapid diagnosis of the disease allows for the implementation of proper treatment and prevention of complications.

Behavioural therapy is an essential part of the treatment of all patients with diabetes mellitus (both type 1 and type 2) of all ages. Proper nutrition and physical activity are crucial for the improvement of patients' general health and the prevention and treatment of chronic complications of diabetes [1].

It is becoming more and more common that a patient with diagnosed diabetes goes to a primary care physician, diabetologist or cardiologist for the purpose of being qualified to practise amateur sports, including diving. The aim of this publication is to review the current recommendations for recreational diving of diabetic patients.

\section{DisCUSSION}

The "Clinical recommendations for therapy of patients with diabetes" issued by the Polish Diabetes Society unequivocally state that physical exercise, due to its multidirectional benefits, is an integral part of a proper comprehensive treatment of diabetes. They indicate that the intensity of physical activity should be determined by the doctor on the basis of the full clinical picture. Potential risks associated with practising sports include the risk of hypoglycaemia, metabolic decompensation and deterioration of the patient's general condition [1]. The recommendations do not specifically address the particular sports practiced by diabetic patients.

According to the "Law on Physical Culture" of January 1996, diving requires appropriate qualifications confirmed by a relevant document and compliance with safety rules. Before commencing a certified diving course, participants are asked to fill in a medical form disclosing information relating to the condition of their general health. Positive answers to questions concerning chronic diseases, including diabetes, diagnosed in the participant do not imply disqualification from participation in the training; they are aimed at ensuring safety during diving and eliminating potential hazards. The final qualification for amateur diving is issued by a doctor, not necessarily by a sports medicine doctor.

For a long time there were no guidelines for the qualification for amateur diving of patients with diabetes, and the regulations between different countries differed significantly. In the mid-1970s, after the tragic accident of a diabetic diver in the UK, a complete ban on diving for diabetic patients was introduced. Complete data on this accident were missing, an autopsy analysis showed that the cause of the diver's death was decompression sickness as well as the presence of a persistent foramen ovale in the heart. There was no clear indication that there was any link between diabetes and death. Nevertheless, the ban was in force until 1992, and was repealed only after a re-examination of the event and after an evaluation of medical events in divers with diabetes who, despite the formal ban, continued to practice this sport.

The UK diving ban for diabetics did not include people who did not require treatment and who were only undergoing non-pharmacological treatment. In all recommendations, the most controversial issues in qualifying for amateur diving concerned patients with diagnosed type 1 diabetes and patients with type 2 diabetes treated with insulin. Previous recommendations of the Australian Diabetes Society of 1994 also clearly indicated that patients with diabetes treated with insulin should not practise diving [3]. Gradually, as a consequence of the results of the conducted research, this approach has completely changed and nowadays the role of stratification of risk and proper education of diabetic patients, who would like to dive amateurly $[4,5]$ is emphasised in the qualification process.

A 1997 study of patients with type 1 diabetes carried out in a hyperbaric chamber, in which no significant differences in the glycaemic profile at the sea level and conditions corresponding to diving at a depth of 27 metres enabled the collection of some very important data [6].

Important data were also obtained from prospective cohort studies conducted among diving patients with diabetes. It was demonstrated that with the use of a predetermined protocol of diabetic patients treated with insulin, they can dive safely, with a low risk of hypoglycaemia and related complications. However, it should be remembered that the study was participated by a selected population. The exclusion criteria included the occurrence of diabetes complications in patients and loss of consciousness or hospitalisation due to severe hypoglycaemia. Most patients were also very well balanced $(\mathrm{HbA} 1 \mathrm{c}<7.5 \%)$, with many of them treated with intensive insulin therapy or a personal insulin pump [7-13].

Based on the results of their research, diabetological and diving societies developed guidelines and recommendations for amateur diving of patients with diabetes $[5,14,15]$. No universally accepted, international guidelines have been developed so far.

The authors of the Australian guidelines [5] postulate a three-stage evaluation process:

1 assessment of the patient's capability to dive;

2. assessment of the scope of diving;

3. control and management of glycaemia on the day of diving.

The proposed criteria for admission to diving are:

1. Assessment of diving capability of a patient with diabetes:

- $\quad$ age: $>18$ years,

- treatment of diabetes: 
$\checkmark \quad$ insulin therapy for more than a year,

$\checkmark$ no significant changes in pharmacotherapy in the previous 3 months,

- $\quad \mathrm{HbA} 1 \mathrm{c} \leq 9.0 \%$.,

- ability to correctly use glycaemia monitoring devices,

- awareness of what the impact on glycaemia is due to: supply of bicarbonates, insulin, physical exercise, ability to modify doses correctly,

- absence of severe hypoglycaemia within the last year, no reported loss of consciousness in the course of hypoglycaemia,

- lack of micro and/or macroangiopathic complications of diabetes,

- initial and then annual assessment by the leading doctor and diabetologist/endocrinologist with the authorisation to examine divers,

- in patients >40 years of age, silent myocardial ischaemia assessment (stress ECG examination).

2. Assessment of the scope of diving:

- prior to the second dive performed on the same day at least an hour break on the surface, following the second dive a prolonged rest period,

- diving with a partner who is not a diabetic and who is informed about the disease and is able to intervene in case of hypoglycaemia,

- prohibition of diving without the possibility of a fast ascent, at depths requiring decompression

- avoidance of dives lasting more than one hour or in circumstances that may provoke hypoglycaemia (icy water, heavy physical effort, etc.),

- having orally administered easily assimilable glucose on the surface and during diving as well as possession of glucagon for parenteral administration on the surface, it is required that the diver's partner is trained in its administration.

3. Control and management of glycaemia on the day of diving:

- glyceamia check 60 minutes, 30 minutes and immediately before diving,

- recommended glycaemic values immediately before diving: $8.3-16.7 \mathrm{mmol} / \mathrm{l}$ (and stable or growing),

- with glycaemia of $6.6-8.3 \mathrm{mmol} / \mathrm{l}$ it is recommended to ingest $15 \mathrm{~g}$ of carbohydrates before diving,

- with glycaemia $<6.6 \mathrm{mmol} / \mathrm{l}$, oral intake of $30 \mathrm{~g}$ of carbohydrates is recommended, diving is allowed only after a check, values permitted for diving are $>8.3 \mathrm{mmol} / \mathrm{l}$ and growing,

- with glycaemia $>16.7 \mathrm{mmol} / \mathrm{l}$ the recommended deferral of diving and control of ketones, with a value $>1.0 \mathrm{mmol} / \mathrm{l}$ it is advisable not to dive on a given dive day,

- $\quad$ recommended glycaemic control for all patients immediately following dive completion,

- $\quad$ each subsequent descent shall be conducted in accordance with the above scheme.
In addition, the proposed recommendations include:

- establishment of an unambiguous signalling of hypoglycaemia under water between the diver with diabetes and his partner (letter L - "low") showed by the diver with the thumb and index finger,

- in the case of hypoglycaemia occurring while under water - immediate ascent, oral glucose intake, exit from the water; if properly trained, a diver with diabetes may take glucose paste under water, but the priority is to return to the surface; the entire process should be assisted by a diving partner,

- $\quad$ in patients treated orally on the day of diving it is recommended to skip sulfonylurea derivatives to minimize the risk of hypoglycaemia,

- avoidance of alcohol within 24 hours before the planned dive,

- proper hydration,

- $\quad$ keeping a glycaemic register during diving and using the obtained data for future dives,

- absence of continuous monitoring systems for glycaemia and insulin pumps during diving.

\section{CONCLUSIONS}

Based on the current state of knowledge, it is possible to perform qualification, a risk assessment and issue recommendations for patients with diabetes who would like to dive amateurly. Current recommendations of the various societies require precise clinical evaluation (admission to amateur diving is granted to patients with well-balanced diabetes, without severe episodes of hypoglycaemia and disease complications) and propose the use of predetermined protocols describing in detail the preparation for diving and the procedure during the dive. The protocol presented in this publication, based on Australian [5], allows diabetic patients to prepare themselves precisely for recreational diving.

Such a systematic approach allows to safely qualify diabetic patients, including those treated with insulin, for amateur diving, as long as they are properly educated and are aware of the potential risks associated with practicing this sport. 


\section{REFERENCES}

1. Zalecenia PTD. 2017 Guidelines on the management of diabetic patients. A position of Diabetes Poland. Clin Diabet. 2017;6, Suppl. A: A1-A80;

2. Rutkowski M, Bandosz P, Czupryniak L, Gaciong Z, Solnica B, Jasiel-Wojculewicz H, et al. Prevalence of diabetes and impaired fasting glucose in Poland--the NATPOL 2011 Study. Diabet Med. 2014 Dec;31(12):1568-71;

3. Hazel J. SCUBA diving: ADS position statement. 1994;

4. Edge CJ, St Leger Dowse M, Bryson P. Scuba diving with diabetes mellitus--the UK experience 1991-2001. Undersea Hyperb Med. 2005 JanFeb;32(1):27-37;

5. Johnson R. Insulin-dependent diabetes mellitus and recreational scuba diving in Australia. Diving Hyperb Med. 2016 Sep;46(3):181-5;

6. Edge CJ, Grieve AP, Gibbons N, O'Sullivan F, Bryson P. Control of blood glucose in a group of diabetic scuba divers. Undersea Hyperb Med. 1997 Sep;24(3):201-7;

7. Adolfsson $\mathrm{P}$, Ornhagen $\mathrm{H}$, Jendle $\mathrm{J}$. The benefits of continuous glucose monitoring and a glucose monitoring schedule in individuals with type 1 diabetes during recreational diving. J Diabetes Sci Technol. 2008 Sep;2(5):778-84;

8. Bonomo M, Cairoli R, Verde G, Morelli L, Moreo A, Grottaglie MD, et al. Safety of recreational scuba diving in type 1 diabetic patients: the Deep Monitoring programme. Diabetes Metab. 2009 Apr;35(2):101-7;

9. Dear Gde L, Pollock NW, Uguccioni DM, Dovenbarger J, Feinglos MN, Moon RE. Plasma glucose responses in recreational divers with insulinrequiring diabetes. Undersea Hyperb Med. 2004 Fall;31(3):291-301;

10. Adolfsson $\mathrm{P}$, Ornhagen $\mathrm{H}$, Jendle $\mathrm{J}$. Accuracy and reliability of continuous glucose monitoring in individuals with type 1 diabetes during recreational diving. Diabetes Technol Ther. 2009 Aug;11(8):493-7;

11. Lormeau B, Sola A, Tabah A, Chiheb S, Dufaitre L, Thurninger O, et al. Blood glucose changes and adjustments of diet and insulin doses in type 1 diabetic patients during scuba diving (for a change in French regulations). Diabetes Metab. 2005 Apr;31(2):144-51;

12. Pollock NW, Uguccioni DM, Dear G, Bates S, Albushies TM, Prosterman SA. Plasma glucose response to recreational diving in novice teenage divers with insulin-requiring diabetes mellitus. Undersea Hyperb Med. 2006 Mar-Apr;33(2):125-33:

13. Harrison D, Lloyd-Smith R, Khazei A, Hunte G, Lepawsky M. Controversies in the medical clearance of recreational scuba divers: updates on asthma, diabetes mellitus, coronary artery disease, and patent foramen ovale. Curr Sports Med Rep. 2005 Oct;4(5):275-81;

14. Jendle J, Adolfsson P, Ornhagen H. Swedish recommendations on recreational diving and diabetes mellitus. Diving Hyperb Med. 2012 Dec;42(4):231-3;

15. Johnson R. A day in the life of a diabetic diver: the Undersea and Hyperbaric Medical Society/Divers Alert Network protocol for diving with diabetes in action. Diving Hyperb Med. 2016 Sep;46(3):181-5.

\section{Daria Kugowska}

Klinika Chorób Wewnętrznych i Diabetologii

10 Wojskowy Szpital Kliniczny z Polikliniką w Bydgoszczy 